\title{
Epidemics and aetiology of hand, foot and mouth disease in Xiamen, China, from 2008 to 2015
}

\author{
S. Z. $\mathrm{HE}^{1} \dagger, \mathrm{M} . \mathrm{Y} \cdot \mathrm{CHEN}^{2,3,4} \dagger, \mathrm{X} \cdot \mathrm{R} \cdot \mathrm{XU}^{1}, \mathrm{Q} \cdot \mathrm{YAN}^{2}, \mathrm{~J} . \mathrm{J} \cdot \mathrm{NIU}^{1}, \mathrm{~W} . \mathrm{H} \cdot \mathrm{WU}^{2,3,4}$, \\ X. S. SU ${ }^{2,3,4}$, S. X. GE $E^{2,3,4 *}$, S. Y. ZHANG ${ }^{2,3,4}$ AND N. S. XIA ${ }^{2,3,4}$ \\ ${ }^{1}$ Xiamen Centre for Disease Control and Prevention, Shengguang Road, Jimei District, Xiamen, China \\ ${ }^{2}$ National Institute of Diagnostics and Vaccine Development in Infectious Diseases, Xiamen University, Xiang'an \\ Campus of Xiamen University, South Xiang'an Road, Xiamen, China \\ ${ }^{3}$ State Key Laboratory of Molecular Vaccinology and Molecular Diagnostics, Xiamen University, Xiang'an \\ Campus of Xiamen University, South Xiang'an Road, Xiamen, China \\ ${ }^{4}$ School of Public Health, Xiamen University, Xiang'an Campus of Xiamen University, South Xiang'an Road, \\ Xiamen, China
}

Received 27 July 2016; Final revision 16 January 2017; Accepted 25 January 2017; first published online 3 April 2017

\section{SUMMARY}

Over the past 8 years, human enteroviruses (HEVs) have caused 27227 cases of hand, foot and mouth disease (HFMD) in Xiamen, including 99 severe cases and six deaths. We aimed to explore the molecular epidemiology of HFMD in Xiamen to inform the development of diagnostic assays, vaccines and other interventions. From January 2009 to September 2015, 5866 samples from sentinel hospitals were tested using nested reverse transcription PCR that targeted the HEV 5' untranslated region and viral protein 1 region. Of these samples, 4290 were tested positive for HEV and the amplicons were sequenced and genotyped. Twenty-two genotypes were identified. Enterovirus 71 (EV71) and coxsackieviruses A16, A6 and A10 (CA16, CA6 and CA10) were the most common genotypes, and there were no changes in the predominant lineages of these genotypes. EV71 became the most predominant genotype every 2 years. From 2013, CA6 replaced CA16 as one of the two most common genotypes. The results demonstrate the vast diversity of HFMD pathogens, and that minor genotypes are able to replace major genotypes. We recommend carrying-out long-term monitoring of the full spectrum of HFMD pathogens, which could facilitate epidemic prediction and the development of diagnostic assays and vaccines.

Key words: Enterovirus, epidemiology, infectious disease epidemiology, surveillance.

\section{INTRODUCTION}

Hand, foot and mouth disease (HFMD) is a common infectious disease worldwide. It is caused by various

\footnotetext{
* Author for correspondence: Dr S. X. Ge, National Institute of Diagnostics and Vaccine Development in Infectious Diseases, Xiamen University, Xiang'an Campus of Xiamen University, South Xiang'an Road, Xiamen, China.

(Email: sxge@xmu.edu.cn)

$\dagger$ These authors contributed equally to this article.
}

human enteroviruses (HEVs), particularly enterovirus 71 (EV71) and coxsackievirus A16 (CA16). HEVs belong to the genus Enterovirus and the family Picornavirus [1]. HEVs are characterised by single positive-sense $7 \cdot 4 \mathrm{~kb}$ genomes with single open reading frames. HEVs are classified into four species (A-D), in accordance with their sequence similarity, genome organisation and biological properties.

Children, particularly those under 5 years, tend to be more susceptible to HFMD than others. In most cases, 
the disease is self-limiting. Symptoms of mild disease generally include fever, skin eruptions on the hands and feet, and vesicular eruptions in the mouth. Most people with only these symptoms readily recover [2]. However, severe cases occasionally occur; they can develop rapidly and can involve central nervous system complications, such as encephalitis, brainstem encephalitis, aseptic meningitis, poliomyelitis-like syndrome and, occasionally, death [3].

The first HFMD case, which occurred in New Zealand in 1957, was caused by CA16. Thereafter, HEVs have been circulating throughout the world. HFMD can spread by direct contact with fluid from blisters, saliva and stools from infected patients [4]. Over the past 20 years, widespread outbreaks have been reported in Asia-Pacific regions [4-12]. The occurrence of HFMD throughout mainland China has received unprecedented attention since the first outbreak in 2008 in the city of Fuyang in Anhui Province. From 2008 to 2012, 6932150 probable cases of HFMD (267942 of which were laboratoryconfirmed cases) and 737 deaths were reported in mainland China [10]. In addition to this nationwide surveillance data, several Chinese cities have reported on their local epidemical investigations of HFMD [13-16]. However, most of these studies were crosssectional or short-term studies that disregarded the minor HEV genotypes associated with HFMD.

The extensive HFMD outbreak in China remains ongoing. However, there have been no investigations of the full spectrum of HFMD pathogens, and no identification of the long-term trends in the circulating HEVs. Thus, the changes in recent years in the epidemiology of the full spectrum of HFMD pathogens in China remain unknown. In this study, the HFMD epidemics from 2008 to 2015 in Xiamen (a coastal city in southeast China with an internationalised port) were investigated, and most of the collected HEVs were identified and genotyped. We aimed to provide comprehensive information on the HFMD epidemics (involving the full spectrum of HFMD pathogens) in Xiamen to facilitate the prevention and control of HFMD epidemics and the development of diagnostic assays and vaccines.

\section{METHODS}

\section{Study area}

Xiamen is a medium-sized city in China with large migrant population from both within China and

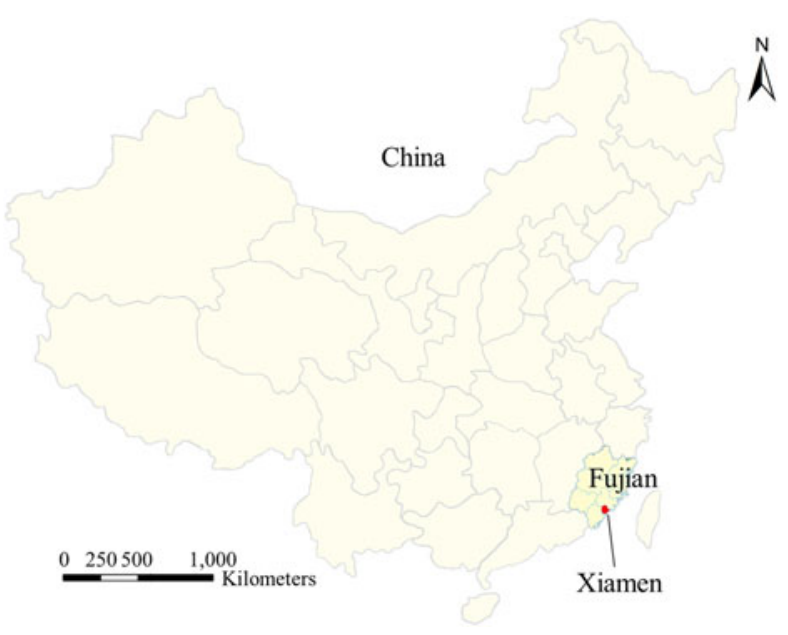

Fig. 1. Location of Xiamen in China.

overseas (Fig. 1). It has a population of 4 million and consists of six administrative districts: Siming, Haicang, Huli, Jimei, Tong'an and Xiang'an. Siming and Huli are commercial urban districts with the highest population density; Haicang, Jimei and Xiang'an are industrial suburban districts where a large number of migrant workers live and work; and Tong'an is a rural district with the lowest population density.

\section{Case definitions and sample collection}

According to the Guidelines on the Diagnosis and Treatment of HFMD issued by the National Health and Family Planning Commission of the People's Republic of China, a clinically diagnosed case is defined as a case involving maculopapular or vesicular eruptions on hands, feet, mouth or buttocks, with or without fever. A laboratory-confirmed case is defined as a HEV case confirmed by nucleotide testing, virus isolation or neutralisation testing. A severe HFMD case is defined as a case with neurological complications (i.e. lethargy, startle, vomiting, coma, limb shaking, acute flaccid paralysis, aseptic encephalitis, aseptic meningitis or brainstem encephalitis) or cardiopulmonary complications (i.e. rapid or slow heart rate, cardiopulmonary failure, myocarditis, pulmonary oedema or pulmonary haemorrhage).

As HFMD has been classified as a class $C$ notifiable infectious disease since 2008, all HFMD cases diagnosed in Xiamen have been reported to the Xiamen Centre for Disease Control and Prevention (CDC). In addition, throat swab samples of the first 10 clinically diagnosed patients who visited the outpatient department at each of the 12 sentinel hospitals in Xiamen were collected every month and sent to the 
Xiamen CDC in sterile viral transport medium. (If there were fewer than 10 patients, all the patients were sampled.) Furthermore, samples from all the severe cases were collected.

Since January 2009, samples have also been sent to our laboratory, the State Key Laboratory of Molecular Vaccinology and Molecular Diagnostics, for aetiological determination. In total, we assessed 5866 specimens between January 2009 and September 2015.

\section{RNA extraction and nested reverse transcription PCR (nRT-PCR)}

The viral RNA was extracted from the samples using GenMagSpin Viral DNA/RNA Isolation Kits (GenMag Biotechnology Co., Ltd., Beijing, China), in accordance with the manufacturer's instructions.

The samples were examined using nRT-PCR, in accordance with our previously reported method [17]. Briefly, the specimens were first tested using sets of broad-spectrum primers for the HEV-5' untranslated region (UTR) and specific primers for the EV71-viral protein 1 (VP1) and CA16-VP1 simultaneously. The samples that were positive for the 5' UTR but negative for EV71-VP1 and CA16-VP1 were then amplified using additional genotype-specific primers for the VP1 region. Finally, all of the amplicons for the VP1 regions were subjected to further sequencing and genotyping. The primers used for genotyping are shown in Supplementary Tables S1 and S2.

\section{Genotyping by phylogenetic analysis}

Multiple sequence alignments were constructed using MEGA software version 5.0 and the Clustal W program. To determine nucleotide similarities, we compared the sequences with standard strains from GenBank (US National Centre for Biotechnology Information).

Phylogenetic trees were constructed using a neighbour-joining cluster algorithm, with evolutionary distances estimated using the Kimura twoparameter model; bootstrapping was performed using 1000 pseudo-replicates [18].

\section{RESULTS} HFMD incidence rates and age and gender distribution
of HFMD cases

From January 2008 to June 2015, 27227 cases of HFMD were reported to the Xiamen CDC,
Table 1. Incidence, severe illness and mortality rates associated with HFMD cases in Xiamen from January 2008 to December 2014

\begin{tabular}{llll}
\hline \hline & $\begin{array}{l}\text { Incidence rate, } \\
\text { per 1 million }\end{array}$ & $\begin{array}{l}\text { Severe illness } \\
\text { rate, per 1 } \\
\text { million }\end{array}$ & $\begin{array}{l}\text { Mortality rate, } \\
\text { per 1 million }\end{array}$ \\
\hline 2008 & $470 \cdot 7$ & $2 \cdot 4$ & 0 \\
2009 & $305 \cdot 2$ & 0 & 0 \\
2010 & $1183 \cdot 1$ & $11 \cdot 6$ & $0 \cdot 3$ \\
2011 & $795 \cdot 6$ & $2 \cdot 8$ & 0 \\
2012 & $918 \cdot 5$ & $2 \cdot 7$ & $0 \cdot 8$ \\
2013 & $1153 \cdot 9$ & $0 \cdot 27$ & 0 \\
2014 & $1713 \cdot 4$ & $2 \cdot 1$ & $0 \cdot 2$ \\
\hline \hline
\end{tabular}

HFMD, hand, foot and mouth disease.

including 99 severe cases and six deaths. The mean HFMD incidence rate from 2008 to 2014 in the general population in Xiamen was $934 \cdot 3 / 1000000$. The annual HFMD incidence rates (Table 1) increased between 2009 and 2014 (from 305.2/1 000000 to $1713 \cdot 4 / 1000000$ ), but the first peak occurred in 2010 , with a rate of $1183 \cdot 1 / 1000000$. During 20082014 , the mean incidence rate of severe cases was $3 \cdot 1 / 1000000$, and the mean mortality rate was $0 \cdot 27 / 1000000$.

The HFMD incidence rate varied considerably with age and gender. Cases involving those aged $<1,1,2,3$, $4,5,6-9$ and $>9$ years accounted for $8 \cdot 83 \%, 27 \cdot 59 \%$, $22 \cdot 51 \%, 18 \cdot 31 \%, 10 \cdot 89 \%, 5 \cdot 45 \%, 4 \cdot 73 \%$ and $1 \cdot 47 \%$ of the total cases, respectively. The highest incidence rate was found in those aged 1 , and children under 5 years accounted for $93.8 \%$ of cases. There were more male patients than female patients, with a male-to-female ratio of $1 \cdot 66: 1$, but there was no difference in the age distribution between male and female patients (Supplementary Fig. S1). The age and gender distributions of the severe cases were similar to those overall.

\section{Temporal distribution of HFMD cases}

The highest number of HFMD cases in Xiamen during the study period was in 2014, and the lowest was in 2009. From 2009 to 2014, with the exception of 2010, the number of HFMD cases increased annually (Fig. 2a). However, $45 \cdot 6 \%$ of the severe cases from 2009 to 2014 were reported in 2010 (Fig. 2b) and $50 \%$ of the deaths from 2009 to 2014 were reported in 2012 . 


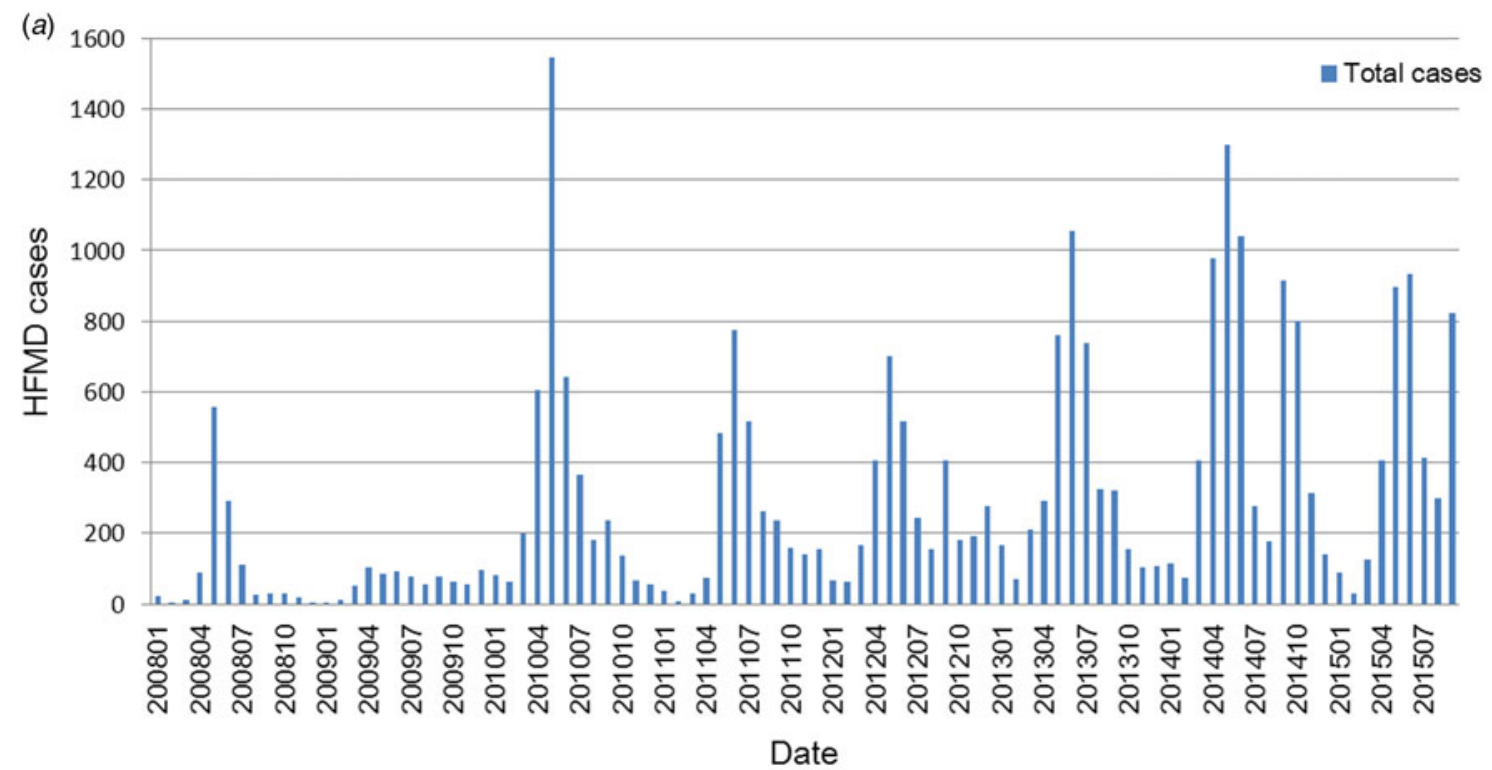

(b)

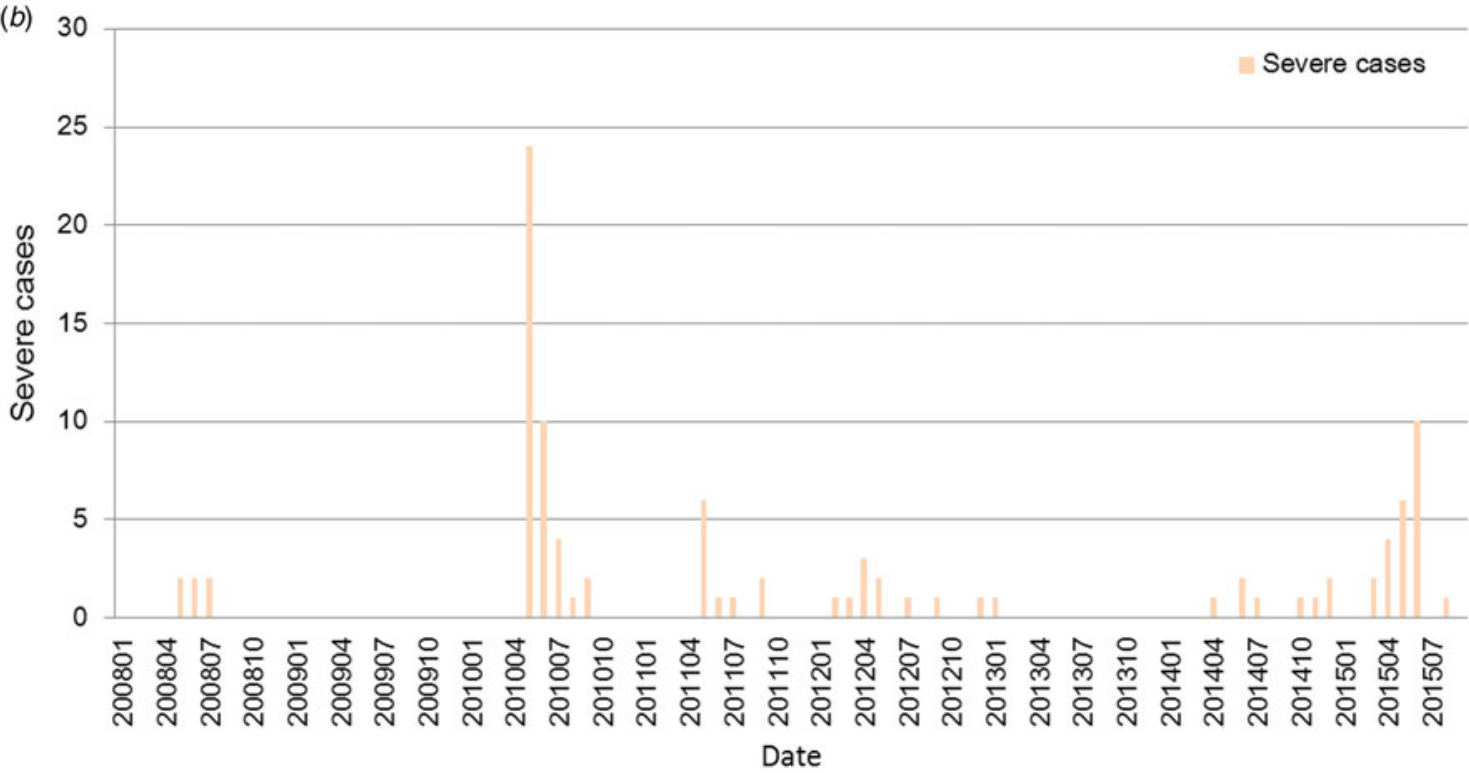

Fig. 2. Temporal distribution of overall and severe HFMD cases in Xiamen from January 2008 to September 2015. (a) Overall and (b) severe HFMD cases by month.

In Xiamen, there was an annual HFMD incidence rate peak in spring and early summer in 2008, 2010, 2011, 2012, 2013, 2014 and 2015, with an additional small peak in the winter of 2012 and an additional large peak in 2014 and 2015. Unlike in the other years, the monthly HFMD incidence rates in 2009 were low and steady, with no peaks (Fig. 2a).

The seasonal distributions were consistent between cases overall and severe cases. Each year, $74.7 \%$ of the severe cases were reported in spring and early summer (Fig. 2b).

\section{Geographical distribution of HFMD cases}

The incidence rate differed greatly between the six districts of Xiamen (Fig. 3). The incidence rates in Haicang, Jimei and Xiang'an were significantly higher than the mean incidence rate, and those in Siming and Huli were below the mean, while Tong'an had the lowest incidence rate of all the districts. However, the incidence rate of severe HFMD cases in Tong'an was significantly higher than the mean, while the incidence rates of severe HFMD cases in Haicang and 


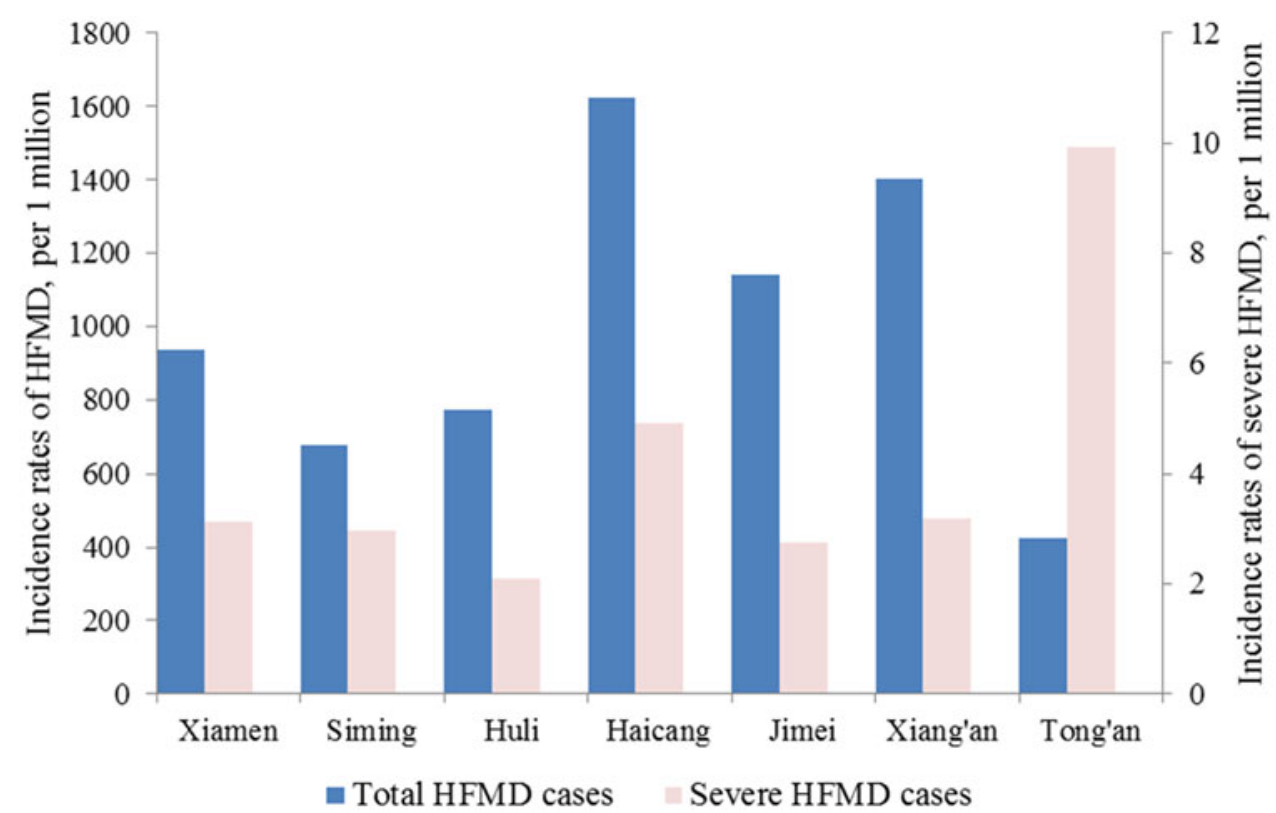

Fig. 3. Annual incidence rates of overall HFMD cases in Xiamen, and Siming, Haicang, Huli, Jimei, Tong'an and Xiang'an districts from 2008 to 2014.

Xiang'an were slightly above those in Siming, Huli and Jimei (Fig. 3).

\section{Clinical manifestations in severe HFMD cases}

For the 99 severe cases and six cases involving deaths, the most common symptoms were fever and maculopapular and vesicular eruptions (Table 2). Central nervous system complications such as convulsions and limb shaking were also common. Some of these cases also involved cardiopulmonary complications.

\section{Pathogen identification}

Since January 2009, throat swab samples from patients with HFMD have been sent to our laboratory, the State Key Laboratory of Molecular Vaccinology and Molecular Diagnostics, for further study. Some specimens were excluded due to lack of background information, such as the patient's age and sex, and the onset of HFMD and sampling dates. In total, we analysed data on 5866 cases, which included 3669 male and 2197 female patients.

An overview of the HFMD cases detected in our laboratory is shown in Supplementary Table S3. The mean age was $2.47 \pm 2.67$ years, and the male-to-female ratio was $1 \cdot 67: 1$, which is consistent with the statistics for the overall HFMD cases in
Xiamen. This suggested that the throat swab samples were a good representation of the cases overall.

Of the 5866 specimens, 4290 were HEV-positive (73.1\%). Most of the HEV genotypes in these HEV-positive cases were identified, except for 167. The majority of pathogens belonged to species A, including EV71, CA16 and coxsackieviruses A6 and A10 (CA6 and CA10). The others belonged to species $\mathrm{B}$, and coxsackievirus B3 (CB3) accounted for the largest proportion.

Our data show that the major HFMD pathogen changed periodically over the study period from January 2009 to September 2015. The full spectrum of HFMD pathogens per quarter is presented in Fig. $4 \mathrm{a}$, and the four most common HEV species in each year (which accounted for $>90 \%$ of HFMD cases in each year) are shown in Table 3. EV71 was always one of the most predominant HEVs (it ranked first in 2010, 2012 and 2014, and second in the other years).

CA6 replaced CA16 from 2012 onwards as one of the top two HEV genotypes. CA16 accounted for $71.8 \%$ of HFMD cases in 2009 , but the proportion decreased over time (it was about $30 \%$ in 2010 and $2011,10-20 \%$ from 2012 to 2014 , and only $6 \cdot 38 \%$ in 2015). Meanwhile, the proportion of CA6 increased from about $3 \%$ in 2009 and 2010 to $20 \%$ in 2011 and 2012, and 59.6\% in 2013 (when it became the most predominant genotype). Although CA10 was 
Table 2. Symptoms of the 99 severe HFMD cases and the six cases involving death

\begin{tabular}{ll}
\hline \hline Symptom & Number of cases $(\%)$ \\
\hline Fever & $89(84 \cdot 76)$ \\
Maculopapular eruptions & $59(56 \cdot 19)$ \\
Vesicular eruptions & $47(44 \cdot 76)$ \\
Limb shaking & $39(37 \cdot 14)$ \\
Convulsions & $23(21 \cdot 90)$ \\
Central nervous system involvement & $24(22 \cdot 86)$ \\
Myoclonic jerk & $9(8 \cdot 57)$ \\
Vomiting & $18(17 \cdot 14)$ \\
Tachypnoea & $18(17 \cdot 14)$ \\
Rapid heart rate & $8(7 \cdot 62)$ \\
Startle & $8(7 \cdot 62)$ \\
Headache & $5(4 \cdot 76)$ \\
Coughing & $6(5 \cdot 71)$ \\
Sore throat & $1(0 \cdot 95)$ \\
Coma & $1(0 \cdot 95)$ \\
Cardiopulmonary failure & $2(1 \cdot 90)$ \\
Aseptic encephalitis & $5(4 \cdot 76)$ \\
Brainstem encephalitis & $1(0 \cdot 95)$ \\
Neurogenetic pulmonary oedema & $2(1 \cdot 90)$ \\
Pulmonary haemorrhage & $3(2 \cdot 86)$ \\
Respiratory and circulatory failure & $2(1 \cdot 90)$ \\
Death & $6(5 \cdot 71)$ \\
\hline \hline
\end{tabular}

HFMD, hand, foot and mouth disease.

never the most predominant genotype, it was always among the four most common HEVs except in 2014. In 2014, CA4, rather than CA10, ranked fourth.

In addition to the four most common HEVs (EV71, CA16, CA6 and CA10), we also detected other HEVs circulating at lower levels (Supplementary Table S4). These pathogens belonged to species A and B. No genotypes belonging to species $\mathrm{C}$ or $\mathrm{D}$ were identified.

For the severe cases, the major HFMD pathogens did not change periodically (Fig. 4b). EV71 was responsible for $78.08 \%$ of the severe cases, CA16 for $4 \cdot 11 \%$, CA10 for $9 \cdot 59 \%$, and other HEVs for $8 \cdot 22 \%$. None of the severe cases were caused by CA6. All the cases involving death were caused by EV71.

There was no significant difference in the age distribution (Supplementary Fig. S2) or male-to-female ratio between the genotype-specific cases and the cases overall. For each genotype, $>70 \%$ of cases were found in children aged $0 \cdot 5-4$ years, i.e. $71 \cdot 9 \%$ of EV71 cases, $88.5 \%$ of CA16 cases, $93.8 \%$ of CA6 cases, $91.4 \%$ of CA10 cases and $86.5 \%$ of the other cases. However, CA6 and CA10 were more likely to affect younger children $(\leqslant 2$ years) than EV71 and CA16 (Supplementary Fig. S2).

\section{Phylogenetic analysis of major pathogens}

Phylogenetic analysis revealed that the predominant lineages of EV71, CA16, CA6 and CA10 did not change from 2009 to 2015 (Supplementary Figs S3S7). Based on the partial VP1 sequences, almost all the EV71 strains clustered in sub-genogroup C4a, though a few strains in the $\mathrm{B} 5, \mathrm{C} 2$ and $\mathrm{C} 5$ genogroups were also identified (Supplementary Fig. S3).

We only performed a phylogenetic analysis of CA16 strains isolated from 2009 to 2012 because these strains caused milder symptoms and there was a significant reduction in CA16 cases from 2013 onwards. Based on the partial VP1 sequences, the CA16 strains were divided into two sub-genogroups, B1a and B1b (Supplementary Fig. S4), and B1b was the predominant lineage (Supplementary Fig. S5).

The complete VP1 sequences of CA6 and CA10 were analysed. The vast majority of CA6 strains clustered in genogroup A, though some strains isolated in 2010-2013 clustered in genogroups B and D (Supplementary Fig. S6). Almost all the CA10 strains belonged to one lineage in genogroup $\mathrm{A}$, though some strains isolated in 2015 belonged to a new lineage in genogroup A (Supplementary Fig. S7).

Phylogenetic analysis of EV71 VP1 sequences showed that strains isolated from severe or mild cases were dispersed in one sub-cluster, with a high degree of homology. Phylogenetic analysis of CA16 and CA10 strains isolated from severe and mild cases led to similar results to those for the EV71 strains.

\section{DISCUSSION}

In addition to the analysis of 2008-2015 data on all the 27227 clinical HFMD cases diagnosed in local hospitals that were reported to Xiamen CDC, almost all the HEV strains collected during 2009-2015 from the sentinel hospitals were successfully identified and genotyped. Based on these data, we were able to comprehensively investigate the HFMD epidemics and the molecular epidemiology of HEVs in Xiamen.

As a result of Xiamen's geographic location and high degree of population mobility, the HFMD epidemic in Xiamen may be similar to or different from other areas of China. The age and gender distributions of HFMD in Xiamen were similar to those in other areas of China $[5,16,19-23]$. However, the temporal distribution in Xiamen exhibited unique characteristics. There was one annual outbreak (peaking in 



Fig. 4. Full spectrum of HFMD pathogens in Xiamen from January 2009 to September 2015. (a) Overall HFMD cases by quarter and $(b)$ severe HFMD cases by year.

June) in northern China, and two annual outbreaks (peaking in May and October) in southern China $[10,24]$. In Xiamen, the temporal distribution was mixed, with one outbreak peak in 2008-2011 and 2013, and two in 2012, 2014 and 2015. In 2012, the first outbreak peak occurred in the spring and early summer, followed by an additional smaller peak in the autumn. However, in 2014 and 2015, after the first large peak in May, another large peak occurred in the autumn. This may be attributable to the combined effects of the climatic, demographic and virus virulence factors in Xiamen.

In addition, although the HFMD incidence rates in southern and eastern China were significantly higher than those in western, northeastern and northern
China [10, 24], the incidence rates in Xiamen were lower than the national rates from 2009 to 2013 (though the 2008 rate in Xiamen was higher than the national rate, while the national rates for 2014 onwards have not yet been reported). This might be because the percentage of susceptible individuals in Xiamen is lower than the national percentage.

Interestingly, the incidence rates in different districts of Xiamen varied. Although the urban districts, Siming and Huli, have much higher population densities and a much higher degree of migration, the incidence rates in these districts were lower than in the other districts, most likely because of their better health systems. Tong'an, the only rural districts in Xiamen, also had a low incidence rate, perhaps 
Table 3. Four most common HEV genotypes identified in HFMD cases in Xiamen from 2009 to 2015

\begin{tabular}{|c|c|c|c|c|}
\hline \multirow{2}{*}{ Year } & \multicolumn{4}{|l|}{ Rank (\%) } \\
\hline & First & Second & Third & Fourth \\
\hline 2009 & CA16 (71·81) & EV71 (14·22) & CA10 $(7 \cdot 14)$ & CA6 $(2 \cdot 88)$ \\
\hline 2010 & EV71 (57.40) & CA16 (32.84) & CA6 $(2 \cdot 47)$ & CA10 $(1 \cdot 08)$ \\
\hline 2011 & CA16 $(33 \cdot 14)$ & EV71 (31.41) & CA6 (21.90) & CA10 (2.88) \\
\hline 2012 & EV71 (52.50) & CA6 (18.93) & CA16 (13.21) & CA10 (5.00) \\
\hline 2013 & CA6 (59.65) & EV71 (17-19) & CA16 (9.82) & CA10 (3.86) \\
\hline 2014 & EV71 (32.05) & CA6 (29.88) & CA16 (17.83) & CA4 (13.0) \\
\hline 2015 & CA6 (40.78) & EV71 (34.04) & CA10 (10.64) & CA16 (6.38) \\
\hline
\end{tabular}

HEV, human enterovirus; HFMD, hand, foot and mouth disease; EV, enterovirus; $\mathrm{C}$, coxsackievirus.

because its lower population density and rate of migration limited the spread of the disease. The industrial suburban districts, Haicang, Xiang'an and Jimei, have large migrant populations and relatively poor sanitary conditions, which resulted in the high incidence rates in these districts. These findings indicate that crowding and poor sanitation are risk factors for HFMD. However, Tong'an had the highest incidence rates of severe HFMD. This could be due to its relatively underdeveloped health system and medical conceptions.

Most previously published epidemiological studies on HFMD conducted in China have been short-term or cross-sectional surveys that have focused only on EV71 and CA16 [25-27]. Therefore, little is known about the long-term epidemical trends of HFMD pathogens in China. In this study, we used a new strategy to detect and genotype the full spectrum of HEVs that cause HFMD (based on our previous work [17]), and we analysed data on the full spectrum of HFMD pathogens that circulated in Xiamen over a period of 7 years. The data indicate that there have been cyclical epidemics involving annual changes in the predominant pathogens, namely, CA16 in 2009 and 2011, EV71 in 2010, 2012 and 2014, and CA6 in 2013 and 2015. A similar phenomenon to that involving the predominance of EV71 every 2 years in Xiamen has been observed in Taiwan, where EV71 epidemics have appeared periodically every 2-3 years [7]. Herd immunity and supplement of susceptible population may explain these patterns.

In 2013 in Xiamen, the proportion of CA16 cases sharply declined (so that CA16 was no longer one of the top two HEVs), while the proportion of CA6 cases rapidly increased. The reason for this is unclear. It cannot be explained simply by herd immunity or altered infectivity due to viral mutations. In this period, the predominant EV71 lineage had been widely circulating for a long time and yet it remained in the top two genotypes. In addition, compared with the predominant CA16 and CA6 lineages isolated before 2013, no characteristic mutations in the predominant CA16 and CA6 lineages were identified since 2013. CA6 has accounted for a growing percentage of HFMD cases in Xiamen in recent years, a phenomenon that has also been observed in many other areas of China [28-32]. Most cases caused by CA6 have been self-limiting, and severe HFMD cases have not been reported among individuals infected with CA6 [20, 33].

Although some EV71 strains belonging to subgenogroups $\mathrm{B} 5, \mathrm{C} 2$ and $\mathrm{C} 5$ were identified, the EV71 sub-genogroup C4a was always the predominant lineage in Xiamen. This is very different from the situation in Taiwan, despite Xiamen and Taiwan being located on opposite sides of the Taiwan Strait, and the high degree of migration between them. In Taiwan, EV71 reoccurred every 2-3 years as the predominant HEV strain, which was always accompanied by a lineage change, e.g. B4 in 2002, C4 in 2004-2005, B5 in 2008-2009, C4 in 2010 and B5 in 2011-2012 [7]. The difference may have occurred because the susceptible population in mainland China is larger than in Taiwan, and so EV71 was under less herd immunity-related selective pressure in mainland China. Moreover, it could be speculated that the $\mathrm{C} 4 \mathrm{a}$ has competitive advantages compared with other EV71 sub-genogroups, such as higher infectivity.

The VP1 sequences of EV71, CA16 and CA10 strains that cause mild and severe disease were highly homologous. Possible reasons for the different clinical 
phenotypes are as follows: (1) nucleotide and amino acid changes in non-VP1 sequences might augment the virulence of HEVs [34, 35] and (2) differences in individual immunity might be the driver of the different clinical manifestations.

Our study indicates that the HEVs associated with HFMD cocirculating in Xiamen are highly diverse, which is likely to be similar to the situation in other areas of mainland China. Besides EV71, CA16 and CA6, we detected five HEV genotypes of species A and 14 of species B (Supplementary Table S4), which had largely been disregarded in most surveillance studies of HFMD-associated HEVs conducted in mainland China. It is possible that one or more HEV species from among the minor circulating pathogens will become predominant in the future, as CA6 did in 2013. In addition, it is noteworthy that some of them, such as CB3 and CA10, can cause severe HFMD. In particular, the proportion of severe cases among CA10-infected patients increased significantly over the course of the study period, and this was accompanied by the occurrence of a new lineage in 2015 (the data have not yet been reported). We recommend that non-EV71 and non-CA16 HEVs should be comprehensively identified, and studies on the molecular epidemiology of HEVs should be conducted as part of routine surveillance of HFMD. This would provide useful epidemical information, and facilitate the development of diagnostic assays and vaccines for HFMD.

In summary, we reported on the HFMD epidemics in Xiamen over the past 8 years, and elucidated the full spectrum of HEVs associated with HFMD and the molecular epidemiology of these HEVs. This is the first long-term longitudinal study on HFMD epidemics involving the identification of HFMD-associated HEVs in a specific administrative region in China. The findings provide comprehensive information for understanding the ongoing HFMD epidemics in mainland China, and will be of value in the development of effective strategies for HFMD prevention and control.

\section{SUPPLEMENTARY MATERIAL}

The supplementary material for this article can be found at https://doi.org/10.1017/S0950268817000309

\section{ACKNOWLEDGEMENTS}

This study was supported by the Natural Science Foundation of Fujian Province (Grant numbers
2013J06016 and 2015J05073); and the Scientific and Technological Innovation Platform of Fujian Province (Grant number 2014Y2004).

\section{DECLARATION OF INTEREST}

None.

\section{REFERENCES}

1. Caro V, et al. Molecular strategy for 'serotyping' of human enteroviruses. Journal of General Virology 2001; 82: 79-91.

2. Ooi MH, et al. Clinical features, diagnosis, and management of enterovirus 71. Lancet Neurology 2010; 9: $1097-1105$.

3. Weng KF, et al. Neural pathogenesis of enterovirus 71 infection. Microbes and Infection 2010; 12: 505-510.

4. Podin Y, et al. Sentinel surveillance for human enterovirus 71 in Sarawak, Malaysia: lessons from the first 7 years. BMC Public Health 2006; 6: 180.

5. Chen SC, et al. An eight-year study of epidemiologic features of enterovirus 71 infection in Taiwan. American Journal of Tropical Medicine and Hygiene 2007; 77: 188-191.

6. Hsu CH, et al. Epidemiologic and clinical features of non-polio enteroviral infections in northern Taiwan in 2008. Journal of Microbiology Immunology and Infection 2011; 44: 265-273.

7. Wu WH, et al. Molecular epidemiology of enterovirus 71 infection in the central region of Taiwan from 2002 to 2012. PLoS ONE 2013; 8: e83711.

8. Chatproedprai $\mathbf{S}$, et al. Clinical and molecular characterization of hand-foot-and-mouth disease in Thailand, 2008-2009. Japanese Journal of Infectious Disease 2010; 63: 229-233.

9. Ang LW, et al. Epidemiology and control of hand, foot and mouth disease in Singapore, 2001-2007. Annals Academy of Medicine Singapore 2009; 38: 106-112.

10. Xing W, et al. Hand, foot, and mouth disease in China, 2008-12: an epidemiological study. Lancet Infectious Disease 2014; 14: 308-318.

11. Wu Y, et al. The largest outbreak of hand, foot and mouth disease in Singapore in 2008: the role of enterovirus 71 and coxsackievirus A strains. International Journal of Infectious Disease 2010; 14: e1076-e1081.

12. Mizuta K, et al. Molecular epidemiology of enterovirus 71 strains isolated from children in Yamagata, Japan, between 1990 and 2013. Journal of Medical Microbiology 2014; 63: 1356-1362.

13. De W, et al. A large outbreak of hand, foot, and mouth disease caused by EV71 and CAV16 in Guangdong, China, 2009. Archives of Virology 2011; 156: 945-953.

14. He YQ, et al. Emergence, circulation, and spatiotemporal phylogenetic analysis of coxsackievirus a6and coxsackievirus a10-associated hand, foot, and mouth disease infections from 2008 to 2012 in Shenzhen, China. Journal of Clinical Microbiology 2013; 51: 3560-3566. 
15. Zhu J, et al. Phylogenetic analysis of enterovirus 71 circulating in Beijing, China from 2007 to 2009. PLoS ONE 2013; 8: e56318.

16. Xu GZ, et al. Epidemiological and etiological characteristics of hand-foot-mouth disease in Ningbo, Zhejiang Province, 2008-2011. Zhonghua Liu Xing Bing Xue Za Zhi 2013; 34: 361-536.

17. Ge S, et al. Specific primer amplification of the VP1 region directed by 5' UTR sequence analysis: enterovirus testing and identification in clinical samples from hand-foot-and-mouth disease patients. Journal of Virological Methods 2013; 193: 463-469.

18. Savolainen-Kopra C, et al. Molecular epidemiology and dual serotype specificity detection of echovirus 11 strains in Finland. Virus Research 2009; 139: 32-38.

19. Lo SH, et al. Clinical and epidemiologic features of coxsackievirus A6 infection in children in northern Taiwan between 2004 and 2009. Journal of Microbiology Immunology and Infection 2011; 44: 252-257.

20. Huang Y, et al. Characterization of severe hand, foot, and mouth disease in Shenzhen, China, 2009-2013. Journal of Medical Virology 2015; 87: 1471-1479.

21. Liu W, et al. Spatiotemporal dynamics of hand-foot-mouth disease and its relationship with meteorological factors in Jiangsu Province, China. PLoS ONE 2015; 10: e0131311.

22. Yu DS, et al. Epidemiological features and pathogenic characteristics of hand, foot and mouth disease in Gansu Province, China during 2008-2012. Bing Du Xиe Bao 2014; 30: 25-32.

23. Liu Y, et al. Detecting spatial-temporal clusters of HFMD from 2007 to 2011 in Shandong Province, China. PLoS ONE 2013; 8: e63447.

24. Liu SL, et al. Comparative epidemiology and virology of fatal and nonfatal cases of hand, foot and mouth disease in mainland China from 2008 to 2014. Reviews in Medical Virology 2015; 25: 115-128.
25. Li W, et al. Epidemiology of childhood enterovirus infections in Hangzhou, China. Virology Journal 2015; 12: 58.

26. Huang $\mathbf{X}$, et al. Epidemiological and etiological characteristics of hand, foot, and mouth disease in Henan, China, 2008-2013. Scientific Reports 2015; 5: 8904.

27. Zhang W, et al. An epidemic analysis of hand, foot, and mouth disease in Zunyi, China between 2012 and 2014. Saudi Medical Journal 2015; 36: 593-598.

28. Zeng H, et al. The epidemiological study of coxsackievirus A6 revealing hand, foot and mouth disease epidemic patterns in Guangdong, China. Scientific Reports 2015; 5: 10550.

29. Lu J, et al. Hand, foot and mouth disease in Guangdong, China, in 2013: new trends in the continuing epidemic. Clinical Microbiology and Infection 2014; 20: O442-O445.

30. Han JF, et al. Hand, foot, and mouth disease outbreak caused by coxsackievirus A6, China, 2013. Journal of Infection 2014; 69: 303-305.

31. Hongyan G, et al. Hand, foot and mouth disease caused by coxsackievirus A6, Beijing, 2013. Pediatric Infectious Disease Journal 2014; 33: 1302-1303.

32. Chen W, et al. Molecular epidemiology of HFMD-associated pathogen coxsackievirus A6 in Fujian Province, 2011-2013. Bing Du Xue Bao 2014; 30: 624-629.

33. Feder HM Jr., Bennett N, Modlin JF. Atypical hand, foot, and mouth disease: a vesiculobullous eruption caused by coxsackie virus A6. Lancet Infectious Disease 2014; 14: 83-86.

34. Zhong Z, et al. Mutations at nucleotides 573 and 579 within 5'-untranslated region augment the virulence of coxsackievirus B1. Virus Research 2008; 135: 255-259.

35. Huang SW, et al. Mutations in VP2 and VP1 capsid proteins increase infectivity and mouse lethality of enterovirus 71 by virus binding and RNA accumulation enhancement. Virology 2011; 422: 132-143. 